\title{
Promoting and Optimizing the Use of 3D-Printed Objects in Spontaneous Recognition Memory Tasks in Rodents: A Method for Improving Rigor and Reproducibility
}

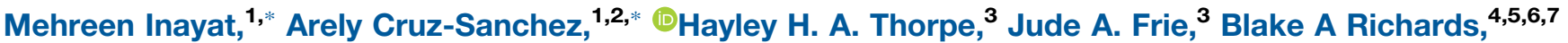 \\ (1) Jibran Y. Khokhar, ${ }^{3}$ and Maithe Arruda-Carvalho ${ }^{1,2}$
}

https://doi.org/10.1523/ENEURO.0319-21.2021

${ }^{1}$ Department of Psychology, University of Toronto Scarborough, Toronto, Ontario M1C1A4, Canada, ${ }^{2}$ Department of Cell and Systems Biology, University of Toronto Scarborough, Toronto, Ontario M1C1A4, Canada, ${ }^{3}$ Department of Biomedical Sciences, Ontario Veterinary College, University of Guelph, Guelph, Ontario N1G2W1, Canada, ${ }^{4}$ Mila, Montréal, Quebec, H2S 3H1, Canada, ${ }^{5}$ Department of Neurology and Neurosurgery, McGill University, Montréal, Quebec, H3A 2B4, Canada, ${ }^{6}$ School of Computer Science, McGill University, Montréal, Quebec, H3A 2A7, Canada, and ${ }^{7}$ Learning in Machines and Brains Program, Canadian Institute for Advanced Research, Toronto, Ontario, M5G 1M1, Canada

\begin{abstract}
Spontaneous recognition memory tasks are widely used to assess cognitive function in rodents and have become commonplace in the characterization of rodent models of neurodegenerative, neuropsychiatric and neurodevelopmental disorders. Leveraging an animal's innate preference for novelty, these tasks use object exploration to capture the what, where and when components of recognition memory. Choosing and optimizing objects is a key feature when designing recognition memory tasks. Although the range of objects used in these tasks varies extensively across studies, object features can bias exploration, influence task difficulty and alter brain circuit recruitment. Here, we discuss the advantages of using 3D-printed objects in rodent spontaneous recognition memory tasks. We provide strategies for optimizing their design and usage, and offer a repository of tested, open-source designs for use with commonly used rodent species. The easy accessibility, low-cost, renewability and flexibility of 3D-printed open-source designs make this approach an important step toward improving rigor and reproducibility in rodent spontaneous recognition memory tasks.
\end{abstract}

Key words: 3D printing; object recognition; open-source; rodents

\section{Significance Statement}

Spontaneous recognition memory tasks are becoming standard in neuroscience labs studying cognitive function and using preclinical models of neurodegenerative, neuropsychiatric and neurodevelopmental disorders. However, variability in object selection across labs may hinder cross-lab comparisons and consensus across the field. Here, we discuss the advantages of, and optimization strategies for, the use of 3Dprinted objects in rodent spontaneous recognition memory tasks, with the goal of increasing accessibility, reproducibility and rigor when running these tasks. We also share tested, open-source object designs for rats and mice with the broader scientific community.

Received July 27, 2021; accepted September 1, 2021; First published September 9, 2021.

The authors declare no competing financial interests.
Author contributions: M.I., A.C.-S., H.H.A.T., J.A.F., B.A.R., J.Y.K., and M.A.-C. designed research; M.I., A.C.-S., H.H.A.T., and J.A.F. performed research; M.I., A.C.-S., H.H.A.T., and J.A.F. analyzed data; B.A.R., J.Y.K., and M.A.-C. wrote the paper. 


\section{Introduction}

Spontaneous recognition memory tasks have become a commonly used tool to assess cognitive function in rodents. These tasks rely on innate novelty preference in rodents to assess recognition memory for object features (novel object recognition; Mathiasen and DiCamillo, 2010; Leger et al., 2013; Lueptow, 2017), spatial location (object location; Vogel-Ciernia and Wood, 2014), recency of events (temporal order recognition; Barker et al., 2007; Barker and Warburton, 2011; Cruz-Sanchez et al., 2020), object category (object category recognition; Creighton et al., 2019), or a combination of features (e.g., object-place, object-in context, object-place-context, multisensory object oddity, object temporal location; Dix and Aggleton, 1999; Ameen-Ali et al., 2015; Cloke et al., 2016; Ramsaran et al., 2016a,b; Barker et al., 2017, 2020). The ability to recognize novel stimuli is critical for survival, emphasizing the ethological relevance of spontaneous recognition memory tasks. Furthermore, the simplicity of these tasks, which part with a need for prior training, food/water restrictions, specialized equipment or stress, makes them extremely accessible and versatile with considerable translational value (Winters et al., 2008; Raber, 2015). As a result, spontaneous object recognition tasks are increasingly being used as cognitive tests in rodent models of neurodegenerative, neuropsychiatric and neurodevelopmental disorders (Lyon et al., 2012; Grayson et al., 2015; Kent et al., 2018; Chini et al., 2020), to improve our understanding of the neural circuit basis of behavior (Winters et al., 2008; Warburton and Brown, 2015), as well as in studies assessing the impact of drugs and toxins on the brain (Dere et al., 2007; Goulart et al., 2010; Nanfaro et al., 2010; Schindler et al., 2010; Hamidullah et al., 2021). Indeed, a PubMed search for "object recognition in rodents" shows a 6-fold increase in publications in the past 15 years, with over 500 publications per year, capturing the continued growth in the popularity of these tasks.

While these advantages have made spontaneous recognition memory tasks a common feature of the behavioral neuroscience repertoire, multiple aspects of the tasks vary considerably between labs, which may hamper consensus and replication in the field. A major source of this variability stems from object selection (Spry et al., 2021). Objects used in spontaneous recognition memory

This work was supported by a Natural Science and Engineering Council of Canada (NSERC) USRA grant (M.I.); the CGS-D Grant CGSD3-534884-2019 (to A.C.-S.); a Vanier award (J.A.F.); Natural Science and Engineering Council of Canada Grants RGPIN-2017-06344 (to M.A.-C.), RGPIN-2019-05121 (to J. Y.K.), and RGPIN-2020-05105 (to B.A.R.); CIFAR (Learning in Machines and Brains Fellowship; B.A.R.), Brain and Behavior Research Foundation (National Alliance for Research on Schizophrenia and Depression Young Investigator 26016); the Canadian Institutes of Health Research (CIHR) Grant PJT 399790); the SickKids Foundation/CIHR-Institute of Human Development, Child and Youth Health Grant NI19-1132R); and the Human Frontier Science Program Organization Grant CDA00009/2018 (to M.A.-C.).

${ }^{*}$ M.I. and A.C.-S. contributed equally to this work.

Correspondence should be addressed to Maithe Arruda-Carvalho at m.arrudacarvalho@utoronto.ca.

https://doi.org/10.1523/ENEURO.0319-21.2021 Copyright @ 2021 Inayat et al.

This is an open-access article distributed under the terms of the Creative Commons Attribution 4.0 International license, which permits unrestricted use, distribution and reproduction in any medium provided that the original work is properly attributed. tasks vary from small plastic toys/parts (Lee et al., 2005; Assini et al., 2009; Heyser and Chemero, 2012; Bath et al., 2017), empty glass or plastic bottles (Eacott and Norman, 2004; Heyser and Chemero, 2012), jars/bottles filled with colored rocks or sand (Sik et al., 2003; Reger et al., 2009; Cyrenne and Brown, 2011; Leger et al., 2013), soda cans (Ramsaran et al., 2016b), candle sticks (Eacott and Norman, 2004; Annie Vogel-Cierna, 2015) to LEGO/ Mega Bloks figures (Hannesson et al., 2004; Norman and Eacott, 2004; Krüger and Hanganu-opatz, 2013; Leger et al., 2013; Lins et al., 2014; Barker and Warburton, 2015; Travaglia et al., 2018). This variability in the objects used introduces additional confounds in the experimental process. Ideally, spontaneous recognition memory tasks would use objects that can be shared across labs.

Luckily, the increased availability and low cost of 3D printing has made it possible for 3D-printed parts to be integrated into a variety of behavioral neuroscience experiments and tools. In our experience, 3D-printed objects using polylactic acid (PLA) or polyethylene terephthalate glycol (PETG) filaments cost approximately USD \$1-3 per printed object depending on object height and fill density used. This has opened the door to many open source tools and apparatuses in the field, such as automated feeding assessment devices (Matikainen-Ankney et al., 2021), automated drinking and lick incidence measurement apparatuses (Frie and Khokhar, 2019; Godynyuk et al., 2019), automated catalepsy measurement tools (Luciani et al., 2020), and capacitance sensor based objects for object recognition (Spry et al., 2021). In the case of spontaneous recognition memory tasks, the use of 3D-printed objects offers the additional advantages of increased design flexibility, uniformity across labs and renewability, thereby increasing rigor, reproducibility and consistency over time. Lastly, consistency in the objects used across institutions could facilitate the creation of large-scale object recognition datasets that could be used to generate hypotheses and test novel questions, similar to what has been done for touch-screen based testing (Beraldo et al., 2019). Here, we provide a perspective on how best to promote and optimize 3D-printed objects for spontaneous recognition memory tests in rodents.

\section{Challenges Associated with Object Selection in Spontaneous Object Recognition Tasks}

One of the major challenges for rodent object recognition tasks is that specific object features inherently affect exploration (Heyser and Chemero, 2012). This is because rodents display innate biases for certain object features, and some object dimensions may induce threatening or anxiogenic elements to the task, particularly in younger rodents (Reger et al., 2009; Cruz-Sanchez et al., 2021). Moreover, the level of similarity between the objects, or feature ambiguity, influences task difficulty and underlying brain circuit recruitment (Norman and Eacott, 2004).

While studies advocate for uniformity in the size and complexity of objects (Silvers et al., 2007), this becomes a challenge when using ready-made/store-bought objects. 
A considerable proportion of spontaneous recognition memory studies do not provide details on the objects used or the baseline preference for these objects, an obstacle for cross-lab comparisons, as well as assertions on feature ambiguity between sampled objects. While the use of LEGO/Mega Bloks figures overcomes some of those barriers, and affords considerable design flexibility, those constructs are still limited in design options and surface area uniformity when compared with 3D-printed objects. Lastly, while touch-screen based objection recognition tasks have also gained popularity (Romberg et al., 2013), and provide valuable translational consistency with tasks used in humans and various options for object optimization, their cost can be prohibitive.

Another issue that presents itself is the additional variability that is introduced when these tasks are used with animal models of neurologic or psychiatric disease, where an animal might display dysfunctions in perception, locomotion, or display anxiety-like behaviors that may cause the animal to stay close to the edges of the arena. These phenotypes may, therefore, impact the animal's ability to perceive or interact with the object features, especially if the objects are not uniform in their appearance. As an example, an animal sticking close to the edge of an open field arena may only interact with the back of an object that has varying features on all its sides, thereby affecting the ability of the animal to assess object novelty, or inducing changes in baseline preference for the object.

\section{The Benefits of Using 3D-Printed Objects}

In recent years, 3D printers have become cheaper and more easily accessible, turning them into a staple at research institutions across the world. Their ubiquity provides an opportunity to increase cross-lab consistency in object selection in spontaneous recognition memory tasks. The flexibility afforded by 3D-printed designs is multi-fold. 3D-printed designs minimize the number of variables driving exploration by matching texture, color, scent, dimensions and surface area across all objects. Changing the filament type can also allow for integration of multi-sensory factors [e.g., using thermoplastic polyurethane (TPU) to create softer objects compared with PLA or acrylonitrile butadiene styrene (ABS)]. Furthermore, objects can achieve any degree of symmetry or subtle manipulations of feature ambiguity, offering ample opportunity for discreet and iterative investigations of object processing. This flexibility is further critical when troubleshooting innate object bias, since any feature driving bias can easily be removed or modified into a new design, and new objects promptly printed for use (see an example in Fig. 1).

Importantly, 3D-printed objects also surmount the challenge of long-term object availability, which often affects continuity when using store-bought objects. Greater uptake of the use of open-source 3D-printed object designs would facilitate comparisons of studies across labs, improving reproducibility and increasing rigor in the interpretation of recognition memory data. Lastly, the relative affordability of these objects could help to improve the uptake of these methods in underfunded research environments.

\section{Strategies for Optimizing the Use of 3D-Printed Objects}

\section{Always test for bias}

As a valuable case study, when one of the authors first started using 3D-printed object designs for novel object recognition, they thought they had been careful because they used designs from another lab. They also made sure to counterbalance objects and sides in their experimental design. Unfortunately, when analyzing their data, they realized that animals showed increased preference when one of the objects was assigned as the novel object, because of innate preference for that object's features, and the data had to be discarded. This cautionary tale emphasizes why excluding the possibility of innate object bias should be the first step when testing any new object combination, and an important analysis to include when publishing data using recognition memory tasks. Importantly, bias should always be assessed in well-handled and habituated animals, as that in itself is a major variable in determining exploration (Powell et al., 2004; Jablonski et al., 2013; Annie Vogel-Cierna, 2015; Cruz-Sanchez et al., 2021).

\section{Mice are not small rats, and neither species is homogenous}

Considerable species (Blaser and Heyser, 2015) and strain (Sik et al., 2003; Ennaceur et al., 2005; Kim et al., 2005; van Goethem et al., 2012) differences in exploration have been reported in spontaneous recognition memory tasks. As different species and strains vary in the way they approach novelty, it is very likely that object combinations that work for a specific species/strain might not translate to another, both in terms of exploration time and bias. As one example, two of the authors' labs had tested and optimized the use of pegs for increasing exploration in C57/129J mice. However, when another author tried these tested designs in rats, they found that the rats chose to climb and bite the pegs instead, and in turn had to optimize completely new object designs (Fig. 2). If a lab is planning to conduct cross-species (or cross-strain) comparisons, testing object combinations should ideally be done in all relevant species/strains from the start, to avoid this scenario. Similarly, while the open-source designs and combinations offered here (Figs. 1, 2; https:// www.thingiverse.com/thing:4964466 and https://www. thingiverse.com/thing:4964541) have worked well within the specified species/strains in tests of novel object recognition, object-location, temporal order recognition (Cruz-Sanchez et al., 2020) and object-place, they must be re-validated before large-scale experiments taking place, especially in novel strains or transgenic animals.

\section{Less is more}

When minimizing bias across objects, a main lesson we have learned is that, counter-intuitively, objects with the highest preference are likely the best ones to avoid. We 

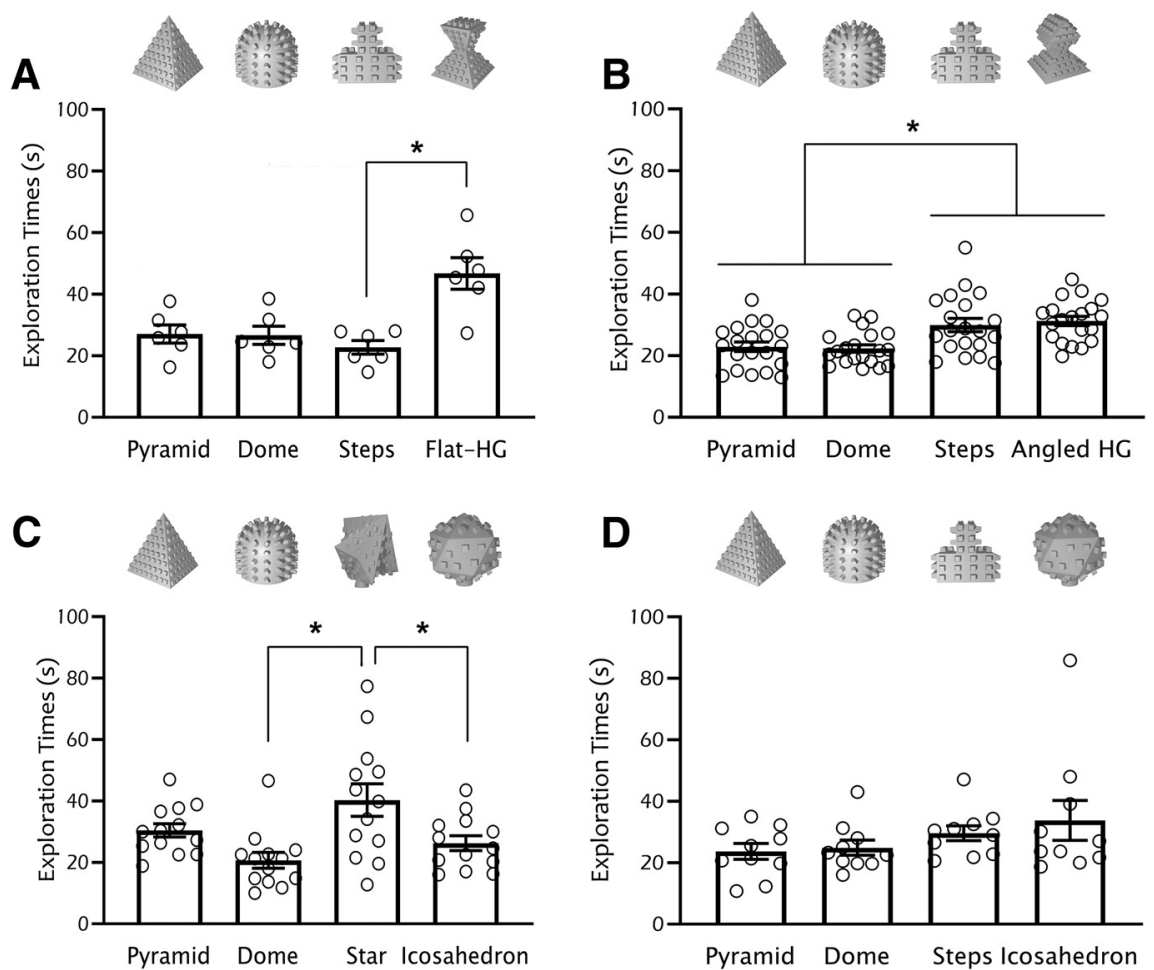

Figure 1. Example data for optimizing the choice of four objects in C57/129J mice. Starting from two previously tested objects (dome and steps), we proceeded to add two new objects with the goal of finding a non-biased four-object combination. When finding innate preference for one or more objects $(\boldsymbol{A})$, possible strategies are to (1) modify features that drive bias $(\boldsymbol{B}$; for example, by removing the flat top in the flat hourglass design); or (2) replace preferred objects until finding an ideal object combination ( $\boldsymbol{C}$; for example, we introduced two novel designs, star and icosahedron, but found innate preference for the star object; this was then followed by $\boldsymbol{D}$, when we replaced the star with the steps object to finally see an absence of innate preference). Multiple rounds of tests may be necessary, but in our experience, innate preferences are quite robust across animal cohorts in future experiments. Bars represent mean exploration time \pm SEM. HG, hourglass; ${ }^{*} p<0.05$. Methodology: adult C57/129J (a cross between C57BLK/6J and 129S1/SvImJ strains) mice were handled and habituated (5 min of handling followed by placement into the behavioral chamber for $4 \mathrm{~min}$ ) to the square open-field behavioral chamber $(30 \times 30 \times 30 \mathrm{~cm})$ twice a day for four consecutive days. On the following day, mice were placed back into the chamber to interact for 5 min with four objects located $3 \mathrm{~cm}$ away from each wall at each corner of the chamber. Exploration time (seconds) per object was measured using ANY-maze software. Object location and start position within the chamber were counterbalanced and randomized across subjects. All animal procedures were approved by the local Animal Care Committee. Statistics: $(\boldsymbol{A})$ oneway RM ANOVA $F_{(3,15)}=7.53, p=0.011$; Tukey's post hoc test shows a significant difference between steps and flat HG $p=0.04 ;(B)$ one-way RM ANOVA $F_{(3,57)}=9.10, p=0.0003$; Tukey's post hoc test shows a significant difference between pyramid versus steps $p=0.042$, pyramid versus angled HG $p=0.0004$, dome versus steps $p=0.031$, dome versus angled HG $p=0.0003 ;(\boldsymbol{C})$ one-way RM ANOVA $F_{(3,36)}=5.59, p=0.003$; Tukey's post hoc test shows a significant difference between dome versus star $p=0.002$, star versus icosahedron $p=0.036 ;(\boldsymbol{D})$ one-way RM ANOVA $F_{(3,27)}=1.51, p=0.25$.

have spent a considerable amount of time designing objects to match the level of innate preference of a particularly preferred object, only to painfully realize it is easier to simply discard a highly preferred object and use two equally, but less preferred ones (Fig. 1). Provided that animals are still spending enough time exploring the objects, in our experience, excluding objects with high innate preference is a more efficient strategy for finding object combinations with equivalent innate preference, as long as any potential floor effects on object preference can be avoided.

\section{Features that can be modified to alter bias}

Many strategies can be employed to optimize object selection and minimize innate bias. The difficulty of choosing object combinations with equivalent innate preference is directly proportional to the number of objects in the task. While optimizing object selection for two-object tasks such as novel object recognition or temporal order recognition may seem like a feasible challenge, this becomes complex for four-object tasks such as objectplace (Fig. 1). Our labs have identified some of the object features that affect exploration. While by no means exhaustive, this list could aid others in employing strategies to optimize 3D-printed object selection in spontaneous recognition memory tasks:

\section{Height of objects}

The height of the object can affect exploration in different ways, relative to the size of the animal. While objects with heights above that of the animal may reduce 
A

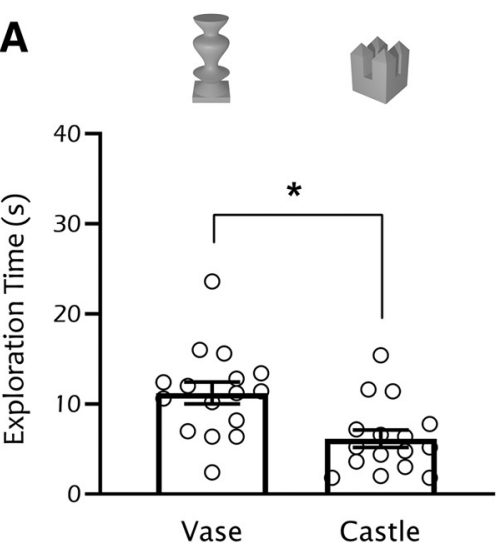

C
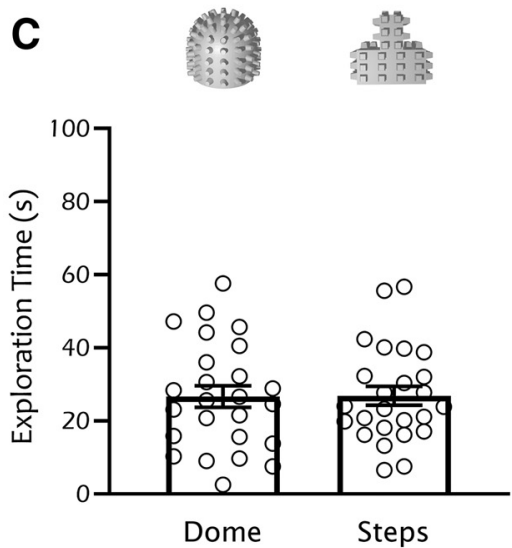

B
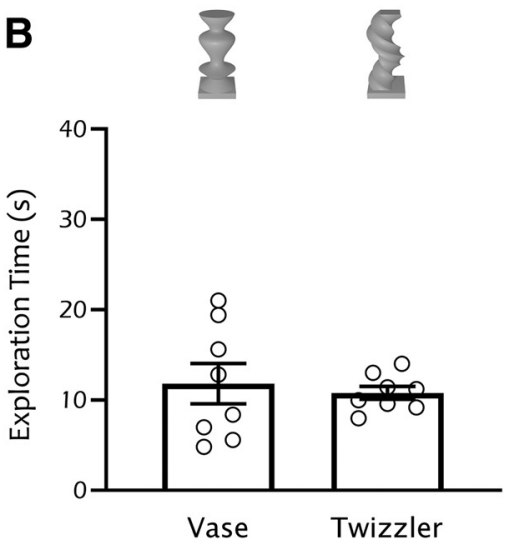

D
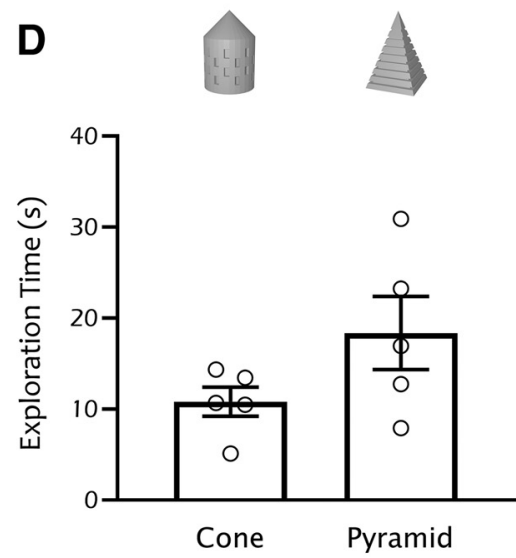

Figure 2. Example data for objects tested in rats, C57/129J and C57BL/6N mice. A, B. Sprague Dawley rats showed a preference for objects closer to their height (vase or twizzler) when compared with smaller objects (castle). $\boldsymbol{C}, \boldsymbol{D}$, Example of two-object combinations tested with $\mathrm{C} 57 / 129 \mathrm{~J}(\boldsymbol{C})$ and C57BL/6N $(\boldsymbol{D})$ mice that showed similar innate preference. Bars represent mean exploration time \pm SEM; ${ }^{*} p<0.05$. Methodology: $(\boldsymbol{A}, \boldsymbol{B})$ adult Sprague Dawley rats were handled for 2 min and habituated to a square open field $(50 \times 50 \mathrm{~cm})$ for $5 \mathrm{~min}$ each day for the $3 \mathrm{~d}$ before experiments. The following day, rats were placed in the open field facing the wall away from the objects with different pairs of objects placed equidistant from each other and the wall $(12.67 \mathrm{~cm})$. Noldus EthoVision software was used to measure cumulative exploration time per object for 5 min with a threshold distance of $2 \mathrm{~cm}$ from the object indicating exploration. Left and right object location within the chamber was counterbalanced. All animal procedures were approved by the local Animal Care Committee. C, Adult C57/129J mice were handled and habituated as described in Figure 1. On the day of testing, mice were placed into the chamber facing the wall located opposite the objects. Objects were placed in the northwest and northeast corners of the chamber, $3 \mathrm{~cm}$ away from each wall. Mice explored the two objects for $5 \mathrm{~min}$. Exploration time (seconds) per object was measured using ANY-maze software. Object location within the chamber was counterbalanced. All animal procedures were approved by the local Animal Care Committee. $\boldsymbol{D}$, Adult C57BL/6N mice were handled for $2 \mathrm{~min} / \mathrm{d}$ across three days before habituation and testing. To habituate mice to the behavioral chamber $(45 \times 45 \times 30 \mathrm{~cm})$, animals were allowed to freely explore the open field for $10 \mathrm{~min} / \mathrm{d}$ across two consecutive days. On the following day, mice were placed back into the open field for 10 min with two unique objects in adjacent corners of the field. Objects were positioned $10 \mathrm{~cm}$ from each wall, and the corner in which each object was placed was counterbalanced across trials. Noldus EthoVision software was used to quantify object exploration with a distance threshold of $2 \mathrm{~cm}$ from the object indicating exploration. All animal procedures were approved by the local Animal Care Committee. Statistics: $(\boldsymbol{A})$ paired $t$ test $t_{(15)}=3.28, p=0.006 ;(\boldsymbol{B})$ paired $t$ test $t_{(7)}=0.49, p=0.36 ;(\boldsymbol{C})$ paired $t$ test $t_{(24)}=0.05, p=0.95 ;(\boldsymbol{D})$ paired $t$ test $t_{(4)}=2.39, p=0.075$.

exploration, likely by inducing anxiety-like behavior (this may particularly affect younger rodents; Reger et al., 2009), if objects are too short they might incite climbing/laying (Fig. 2A). A few developmental studies have proposed to scale the size of objects according to animal size (Ricceri et al., 2000; Reger et al., 2009; Travaglia et al., 2018), something easily achievable with $3 \mathrm{D}$-printed designs. Our data with rats suggest this may also be applicable across species, with rats seemingly preferring taller objects (Fig. 2A). While, to the best of our knowledge, this scaling strategy has not been directly investigated, we expect object height would affect exploration rates across ages and species/strains if objects are too tall or too short. While we have not directly tested variations in object volume, we hypothesize the same principle would apply, and recommend selecting object dimensions that are similar to animal size. 


\section{Protrusions and indentations}

Introduction of concave or convex elements to 3Dprinted objects may affect exploration. We have found that C57/129J mice display a preference for protrusions (e.g., pegs), which increase object exploration in this strain. In contrast, rats climb on pegs instead of exploring the objects. We have found no increase in cleanup time when using pegged objects, provided objects are properly sprayed in between trials.

\section{Flat tops}

In our experience, C57/129J mice tend to lay considerably more on 3D-printed objects with a large flat top, when compared with objects with an angled or curved top. Anecdotally, the object with the most preference two of our labs have found for this strain was a cube with pegs, which substantially exceeded all other tested objects in preference, and was therefore never used. From this and other observations, we try and minimize using objects with large flat surfaces and square angles when working with this strain.

\section{Technical recommendations}

When 3D printing objects for rodent object recognition tasks, we recommend using the same filament (we have tested PLA and PETG filaments) and color for all printed objects, to minimize variables. We have not directly tested whether changes in texture from filament type affect object exploration, but saw similar exploration levels between PLA and PETG filament printed objects. If using automated tracking software, choosing a filament color with the most contrast with the animal coat color will improve tracking. Our labs have used different strategies for immobilizing objects in the arena. Importantly, properly immobilizing objects will minimize any variability in exploration because of object weight, since all objects will be equally immovable by the animal. One option is to use an arena with a metallic floor and attach magnets to the 3Dprinted object (Cruz-Sanchez et al., 2020). This allows flexibility in the location of the objects, while securing the objects in place throughout trials. Another useful alternative that can be used with a variety of surfaces and materials is $3 \mathrm{M}$ Dual Lock strips. These strips can be attached to the arena and objects, locking them in place. Importantly, Dual Lock strips are able to maintain adhesion to surfaces through cage washes, and the objects can be used interchangeably between various arenas. Finally, sticky tack can also be used to immobilize objects. Sticky tack allows for a smaller gap between the object and the floor when compared with the Dual Lock strips, is reusable and easily accessible. However, while sticky tack was strong enough to hold objects in place for tasks with $\mathrm{C} 57 \mathrm{BL} / 6 \mathrm{~N}$ mice, it is not recommended for recognition tasks using rats.

\section{Conclusion}

With the increasing popularity of spontaneous object recognition task variants to assess memory in rodents, approaches that help to standardize these tasks will help improve rigor and reproducibility. Here, we present a case for the use of 3D-printed object designs, and provide a framework for assessing the suitability of these objects across species and task variations. Moreover, through providing validated designs for 3D-printed objects, we hope to promote the harmonization of approaches and methods toward more generalizability of spontaneous object recognition findings.

\section{References}

Ameen-Ali KE, Easton A, Eacott MJ (2015) Moving beyond standard procedures to assess spontaneous recognition memory. Neurosci Biobehav Rev 53:37-51.

Annie Vogel-Cierna MW (2015) Examining object location and object recognition memory in mice. Curr Protoc Neurosci 69:8.31.18.31.17.

Assini FL, Duzzioni M, Takahashi RN (2009) Object location memory in mice: pharmacological validation and further evidence of hippocampal CA1 participation. Behav Brain Res 204:206-211.

Barker GRI, Warburton EC (2011) Evaluating the neural basis of temporal order memory for visual stimuli in the rat. Eur $\mathrm{J}$ Neurosci 33:705-716.

Barker GRI, Warburton EC (2015) Object-in-place associative recognition memory depends on glutamate receptor neurotransmission within two defined hippocampal-cortical circuits: a critical role for AMPA and NMDA receptors in the hippocampus, perirhinal, and prefrontal cortices. Cereb Cortex 25:472-481.

Barker GRI, Bird F, Alexander V, Warburton EC (2007) Recognition memory for objects, place, and temporal order: a disconnection analysis of the role of the medial prefrontal cortex and perirhinal cortex. J Neurosci 27:2948-2957.

Barker GRI, Banks PJ, Scott H, Ralph GS, Mitrophanous KA, Wong L, Bashir ZI, Uney JB, Warburton EC (2017) Separate elements of episodic memory subserved by distinct hippocampal - prefrontal connections. Nat Neurosci 20:242-250.

Barker GR, Wong LF, Uney JB, Warburton EC (2020) CREB transcription in the medial prefrontal cortex regulates the formation of long-term associative recognition memory. Learn Mem 27:45-51.

Bath KG, Nitenson AS, Lichtman E, Lopez C, Chen W, Gallo M, Goodwill H, Manzano-Nieves G (2017) Early life stress leads to developmental and sex selective effects on performance in a novel object placement task. Neurobiol Stress 7:57-67.

Beraldo FH, Palmer D, Memar S, Wasserman DI, Lee WV, Liang S, Creighton SD, Kolisnyk B, Cowan MF, Mels J, Masood TS, Fodor C, Al-Onaizi MA, Bartha R, Gee T, Saksida LM, Bussey TJ, Strother SS, Prado VF, Winters BD, et al. (2019) MouseBytes, an open-access high-throughput pipeline and database for rodent touchscreen-based cognitive assessment. Elife 8:e49630.

Blaser R, Heyser C (2015) Spontaneous object recognition: a promising approach to the comparative study of memory. Front Behav Neurosci 9:183-112.

Chini M, Pöpplau JA, Lindemann C, Carol-Perdiguer L, Hnida M, Oberländer V, Xu X, Ahlbeck J, Bitzenhofer SH, Mulert C, Hanganu-Opatz IL (2020) Resolving and rescuing developmental miswiring in a mouse model of cognitive impairment. Neuron 105:60-74.e7.

Cloke JM, Nguyen R, Chung BYT, Wasserman DI, De Lisio S, Kim JC, Bailey CDC, Winters BD (2016) A novel multisensory integration task reveals robust deficits in rodent models of schizophrenia: converging evidence for remediation via nicotinic receptor stimulation of inhibitory transmission in the prefrontal cortex. J Neurosci 36:12570-12585.

Creighton SD, Collett HA, Zonneveld PM, Pandit RA, Huff AE, Jardine KH, McNaughton BL, Winters BD (2019) Development of an "object category recognition" task for mice: involvement of muscarinic acetylcholine receptors. Behav Neurosci 133:527-536. 
Cruz-Sanchez A, Dematagoda S, Ahmed R, Mohanathaas S, Odenwald N, Arruda-Carvalho M (2020) Developmental onset distinguishes three types of spontaneous recognition memory in mice. Sci Rep 10:10612.

Cruz-Sanchez A, Wilkin J, Arruda-Carvalho M (2021) Ontogeny of spontaneous recognition memory in rodents. Neurobiol Learn Mem 177:107361.

Cyrenne DLM, Brown GR (2011) Ontogeny of sex differences in response to novel objects from adolescence to adulthood in listerhooded rats. Dev Psychobiol 53:670-676.

Dere E, Huston JP, Silva MADS (2007) The pharmacology, neuroanatomy and neurogenetics of one-trial object recognition in rodents. Neurosci Biobehav Rev 31:673-704.

Dix SL, Aggleton JP (1999) Extending the spontaneous preference test of recognition: evidence of object-location and object-context recognition. Behav Brain Res 99:191-200.

Eacott MJ, Norman G (2004) Integrated memory for object, place, and context in rats: a possible model of episodic-like memory? $\mathrm{J}$ Neurosci 24:1948-1953.

Ennaceur A, Michalikova S, Bradford A, Ahmed S (2005) Detailed analysis of the behavior of Lister and Wistar rats in anxiety, object recognition and object location tasks. Behav Brain Res 159:247266.

Frie JA, Khokhar JY (2019) An open source automated two-bottle choice test apparatus for rats. HardwareX 5:e00061.

Godynyuk E, Bluitt MN, Tooley JR, Kravitz AV, Creed MC (2019) An open-source, automated home-cage sipper device for monitoring liquid ingestive behavior in rodents. eNeuro 6:ENEURO.029219.2019.

Goulart BK, de Lima MNM, de Farias CB, Reolon GK, Almeida VR, Quevedo J, Kapczinski F, Schröder N, Roesler R (2010) Ketamine impairs recognition memory consolidation and prevents learninginduced increase in hippocampal brain-derived neurotrophic factor levels. Neuroscience 167:969-973.

Grayson B, Leger M, Piercy C, Adamson L, Harte M, Neill JC (2015) Assessment of disease-related cognitive impairments using the novel object recognition (NOR) task in rodents. Behav Brain Res 285:176-193.

Hamidullah S, Lutelmowski CD, Creighton SD, Luciani KR, Frie JA, Winters BD, Khokhar JY (2021) Effects of vapourized THC and voluntary alcohol drinking during adolescence on cognition, reward, and anxiety-like behaviours in rats. Prog Neuropsychopharmacol Biol Psychiatry 106:110141.

Hannesson DK, Howland JG, Phillips AG (2004) Interaction between perirhinal and medial prefrontal cortex is required for temporal order but not recognition memory for objects in rats. J Neurosci 24:4596-4604.

Heyser CJ, Chemero A (2012) Novel object exploration in mice: not all objects are created equal. Behav Processes 89:232238.

Jablonski SA, Schreiber WB, Westbrook SR, Brennan LE, Stanton ME (2013) Determinants of novel object and location recognition during development. Behav Brain Res 256:140150.

Kent BA, Heath CJ, Kim CH, Ahrens R, Fraser PE, St George-Hyslop P, Bussey TJ, Saksida LM (2018) Longitudinal evaluation of TauP301L transgenic mice reveals no cognitive impairments at 17 months of age. Brain Behav 8:e00896.

Kim D, Chae S, Lee J, Yang H, Shin HS (2005) Variations in the behaviors to novel objects among five inbred strains of mice. Genes Brain Behav 4:302-306.

Krüger H, Hanganu-opatz IL (2013) Neonatal cholinergic lesion alters the acoustic structure of infant rat vocalization but not the early cognitive development. Dev Psychobiol 55:294308.

Lee I, Hunsaker MR, Kesner RP (2005) The role of hippocampal subregions in detecting spatial novelty. Behav Neurosci 119:145-153.

Leger M, Quiedeville A, Bouet V, Haelewyn B, Boulouard M, Schumann-bard P, Freret T (2013) Object recognition test in mice. Nat Protoc 8:2531-2537.
Lins BR, Ballendine SA, Howland JG (2014) Altered object exploration but not temporal order memory retrieval in an object recognition test following treatment of rats with the group II metabotropic glutamate receptor agonist LY379268. Neurosci Lett 560:41-45.

Luciani KR, Frie JA, Khokhar JY (2020) An open source automated bar test for measuring catalepsy in rats. eNeuro 7:ENEURO.048819.2020.

Lueptow LM (2017) Novel object recognition test for the investigation of learning and memory in mice. J Vis Exp 2017:1-9.

Lyon L, Saksida LM, Bussey TJ (2012) Spontaneous object recognition and its relevance to schizophrenia: a review of findings from pharmacological, genetic, lesion and developmental rodent models. Psychopharmacology (Berl) 220:647-672.

Mathiasen J, DiCamillo A (2010) Novel object recognition in the rat: a facile assay for cognitive function. Curr Protoc Pharmacol Chapter 5:Unit 5.59.

Matikainen-Ankney BA, Earnest T, Ali M, Casey E, Wang JG, Sutton AK, Legaria AA, Barclay KM, Murdaugh LB, Norris MR, Chang $\mathrm{YH}$, Nguyen KP, Lin E, Reichenbach A, Clarke RE, Stark R, Conway SM, Carvalho F, Al-Hasani R, McCall JG, et al. (2021) Itle: an open-source device for measuring food intake and operant behavior in rodent home-cages. Elife 10: e66173.

Nanfaro F, Cabrera R, Bazzocchini V, Laconi M, Yunes R (2010) Pregnenolone sulfate infused in lateral septum of male rats impairs novel object recognition memory. Pharmacol Rep 62:265-272.

Norman G, Eacott MJ (2004) Impaired object recognition with increasing levels of feature ambiguity in rats with perirhinal cortex lesions. Behav Brain Res 148:79-91.

Powell SB, Geyer MA, Gallagher D, Paulus MP (2004) The balance between approach and avoidance behaviors in a novel object exploration paradigm in mice. Behav Brain Res 152:341-349.

Raber J (2015) Novel images and novel locations of familiar images as sensitive translational cognitive tests in humans. Behav Brain Res 285:53-59.

Ramsaran Al, Sanders HR, Stanton ME (2016a) Determinants of object-in-context and object-place-context recognition in the developing rat. Dev Psychobiol 58:883-895.

Ramsaran Al, Westbrook SR, Stanton ME (2016b) Ontogeny of object-in-context recognition in the rat. Behav Brain Res 298:37-47.

Reger ML, Hovda DA, Giza CC (2009) Ontogeny of rat recognition memory measured by the novel object recognition task. Dev Psychobiol 51:672-678.

Ricceri L, Colozza C, Calamandrei G (2000) Ontogeny of spatial discrimination in mice: a longitudinal analysis in the modified openfield with objects. Dev Psychobiol 37:109-118.

Romberg C, Horner AE, Bussey TJ, Saksida LM (2013) A touch screen-automated cognitive test battery reveals impaired attention, memory abnormalities, and increased response inhibition in the TgCRND8 mouse model of Alzheimer's disease. Neurobiol Aging 34:731-744.

Schindler AG, Li S, Chavkin C (2010) Behavioral stress may increase the rewarding valence of cocaine-associated cues through a dynorphin/-opioid receptor-mediated mechanism without affecting associative learning or memory retrieval mechanisms. Neuropsychopharmacology 35:1932-1942.

Sik A, Van Nieuwehuyzen P, Prickaerts J, Blokland A (2003) Performance of different mouse strains in an object recognition task. Behav Brain Res 147:49-54.

Silvers JM, Harrod SB, Mactutus CF, Booze RM (2007) Automation of the novel object recognition task for use in adolescent rats. $J$ Neurosci Methods 166:99-103.

Spry KP, Fry SA, Defillip JMS, Drye SG, Stevanovic KD, Hunnicutt J, Bernstein BJ, Thompson EE, Cushman JD (2021) 3D-printed capacitive sensor objects for object recognition assays. eNeuro 8: ENEURO.0310-20.2020. 
Travaglia A, Steinmetz AB, Miranda JM, Alberini CM (2018) Mechanisms of critical period in the hippocampus underlie object location learning and memory in infant rats. Learn Mem 25:176-182.

van Goethem NP, Rutten K, van der Staay FJ, Jans LAW, Akkerman S, Steinbusch HWM, Blokland A, van't Klooster J, Prickaerts J (2012) Object recognition testing: rodent species, strains, housing conditions, and estrous cycle. Behav Brain Res 232:323-334.
Vogel-Ciernia A, Wood M (2014) Examining object location and object recognition memory in mice. Curr Protoc Neurosci 69:8.31.18.31.17.

Warburton EC, Brown MW (2015) Neural circuitry for rat recognition memory. Behav Brain Res 285:131-139.

Winters BD, Saksida LM, Bussey TJ (2008) Object recognition memory: neurobiological mechanisms of encoding, consolidation and retrieval. Neurosci Biobehav Rev 32:1055-1070. 